\title{
Effects of crocin on locomotor activity as well as novel object recognition and object location memories in chronic restraint stressed rats
}

\author{
Maryam Radahmadi*iD , Azadehalsadat Hosseini Dastgerdi, Ali Asghar Pourshanazari \\ Department of Physiology, School of Medicine, Isfahan University of Medical Sciences, Isfahan, Iran
}

\section{Abstract}

Introduction: Chronic stress impairs memory and certain brain functions such as locomotor activity. Crocin is one of the active components of saffron and has neuroprotective effects on brain functions. This study investigated crocin effects on locomotor activity and recognition of new conditions (exploration time) as well as novel object recognition and object location memories in chronic restraint stress rats.

Methods: Thirty-two male Wistar rats were randomly allocated to control group, restraint stress group (6h/day for 21days) and two groups receiving daily intraperitoneal injections of crocin (30 and $60 \mathrm{mg} / \mathrm{kg}$ ) accompanied by restraint stress. Memories were evaluated using the relevant novel object recognition (NOR) and object location (OLT) tests.

Results: The NOR and OLT results, respectively, revealed significant and nonsignificant decreases in locomotor activity in the stressed group. The NOR results revealed enhanced locomotor activity due to crocin administration (30 and 60mg/kg). The NOR revealed significant enhancements in recognizing new conditions in both crocin treatments while the OLT test did so only with a crocin dose of $60 \mathrm{mg} / \mathrm{kg}$. Restraint stress and crocin treatments led to no significant differences in novel object recognition and object location memories. Finally, the stressed group exhibited significant increases in serum corticosterone levels but corticosterone levels declined significantly with crocin dose of $30 \mathrm{mg} / \mathrm{kg}$.

Conclusion: The high and low doses of crocin had different effects on the NOR and OLT variables under restraint stress conditions. The NOR test as cognitive test was found more sensitive to crocin treatments than the OLT test as spatial test although neither the memories showed changes in response to such treatment.

http://dx.doi.org/10.32598/ppj.24.2.80

\section{Keywords:}

Crocin;

Memory impairment;

Restraint stress;

Rat

\section{* Corresponding author: Maryam Radahmadi Email: \\ m_radahmadi@med.mui.ac.ir Tel: +98 (31) 37929176}

Received 25 August 2019; Received in revised form 15 March 2020; Accepted 23 March 2020

\section{Introduction}

Stress, with likely harmful effects on both behavior and physiological systems, reveals itself as physiological and/or psychological responses in response to adverse stimuli (Bandegi et al., 2014; Blanchard et al., 2001). Although stress is an essential requirement for survival, its chronic form might disturb brain functions 
(such as memory processing) by activating the hypothalamus-pituitary-adrenal axis and secreting glucocorticoid hormones (Mohammadi et al., 2014; Sandi and Pinelo-Nava, 2007). In addition, the steroid receptors are highly expressed in the brain structures involved in mental and cognition health (Ghadrdoost et al., 2011).

Crocin (along with picrocrocin and safranal) is one of the major biologically active components of Crocus sativus L. (saffron) (Ghadrdoost et al., 2011) cultivated in various parts of the world including Iran, China, Spain, India and Greece (Pitsikas et al., 2008). It is reported that pharmaceutical advantages as antioxidant and neuroprotective effects, crocin has been found instrumental in improving scopolamine or ethanol-induced impairments of learning and memory as well as brain deficit in rodents (Gonda et al., 2012; Hosseinzadeh and Ziaei, 2006; Pitsikas and Sakellaridis, 2006; Pitsikas et al., 2007). Moreover, high doses of crocin have been shown to exhibit better protective effects on cognitive memory deficit induced by chronic isolation stress that had impaired cognitive and spatial memories (Khani et al., 2018). McMorran et al. (2006) indicated that different types of stress seem to play different roles in impairing different brain functions such as mood, memory, attention and cognition. Hence, more specifically, it is necessary a study strives to determine whether or not different doses of crocin have identical effects on the various aspects of brain functions under different psychological stress types. Therefore, the present study was designed to evaluate the effects of different crocin doses on locomotor activity, recognition of new conditions, novel object recognition and object location memories as well as serum corticosterone level following chronic restraint stress in male rats.

\section{Materials and methods}

\section{Experimental design}

Experiments were performed on 32 male Wistar rats (Pasteur Institute, Tehran, Iran) with initial weights of 250-300g. The rats were maintained under lightcontrolled conditions (12h light/dark; lights on 07:0019:00) in a room with a temperature of $23 \pm 2^{\circ} \mathrm{C}$. Food and water were made available ad libitum, except when the rats were exposed to stress. The study was approved by Committee on Ethics of Animal Use, Isfahan University of Medical Sciences (IR.MUI.REC.1394.3.933) and all the experiments were executed in accordance with the National Institute of Health Guide for the Care and Use of Laboratory Animals (NIH Publications No. 80-23, Rev. 2011)

The rats were randomly divided into the following four groups ( $\mathrm{n}=8$ in each group): control (Co, receiving saline as vehicle daily for 21 days); chronic restraint stress (St.R, stressed in a restrainer $6 \mathrm{~h} /$ day for 21 days) and two groups receiving daily crocin doses (30 and $60 \mathrm{mg} / \mathrm{kg}$ ) accompanied by a period of 21 days of restraint stress (St.R-C30 and St.R-C60).

At the end of the experiments, all the experimental groups were subjected to behavioral tests between 14:00 and 16:00. The animals were evaluated not only in terms of their locomotor activity and recognition of novel conditions but also for their novel object recognition and location memories as judged by the novel object location (OLT) and novel object recognition (NOR) tests, respectively. Serum corticosterone levels were also determined after decapitation.

\section{Drugs}

Crocin was purchased from Sigma-Aldrich Company (USA) in powder form, dissolved in a physiological saline and injected intraperitoneally (IP) at the doses of 30 and $60 \mathrm{mg} / \mathrm{kg}$ daily for 21 consecutive days in the stressed groups prior to exposure to stress (Dastgerdi et al., 2017).

\section{Stress paradigms}

In the current study, rats were placed in restrainers for $6 \mathrm{~h} /$ day (08:00-14:00) for 21 days (Ranjbar et al., 2016). Restraint was defined as a strong stressor in rats to evoke unconditioned and unavoidable neuroendocrine responses (Ranjbar et al., 2016). This type of stress is commonly used in studies of physical and psychological stress (Hosseinzadeh et al., 2004; Patchev and Patchev, 2006; Wu et al., 2016).

\section{Behavioral apparatus and method}

The novel object recognition and object location tests are widely used for assessing memory in both humans and rodents (Ainge et al., 2006; Brodziak et al., 2014; Clark et al., 2000; Ranjbar et al., 2016) and used to evaluate not only the two different cognitive and spatial memories without external motivation but also the degrees of stress in animal experiments 
(Roozendaal et al., 2007; Silvers et al., 2007). In the current study, the NOR and OLT were performed in full compliance with earlier studies (Khani et al., 2018 ; Ranjbar et al., 2016). Prior to the tests, each rat would be individually placed on day 20 of the experimental period in the open field box $(60 \times 60 \times 50 \mathrm{~cm})$ for $5 \mathrm{~min}$ for habituation. The sample and test phases of the OLT and NOR tests would then be performed on day 21 (i.e., the final day of the experiment following the last treatment and the last exposure to stress)(Drumond et al., 2012; Ranjbar et al., 2016).

The times spent to explore novel and familiar locations over a 5-min observation period are designated by the initials $N$ and $F$ in the OLT while these same letters in the NOR test represent the times spent to explore novel and familiar objects (Ranjbar et al., 2016). Locomotor activity in the testtrial phases of both tests was measured as the total time spent exploring each of the two objects (i.e., $\mathrm{T}_{2}=\mathrm{F}+\mathrm{N}$ in the test phase).

Finally, two main and auxiliary discrimination indexes (D1 and D2) were considered as index measures of discrimination between new and familiar objects. Indeed, D1 (the discrimination index; D1= N-F(s)) represents the absolute difference between new and familiar objects, whereas D2 (the discrimination ratio; $\left.D_{2}=[N /(N+F)] \times 100\right)$ is the relative measure of discrimination that corrects D1 for the level of exploratory activity in the test-trial (Akkerman et al., 2012; Ranjbar et al., 2016; Sik et al., 2003; Waters et al., 2012).

\section{Assessment of serum corticosterone levels}

On day 22, at the end of the experimental period, the rats were anesthetized using IP injections of $1.5 \mathrm{~g} / \mathrm{kg}$ urethane before they were sacrificed at 14:00-16:00. Following decapitation, serum was separated from blood samples and stored at $-80^{\circ} \mathrm{C}$ until analysis. Serum corticosterone level was measured in each case using the commercial enzyme-linked immunosorbent assay (ELISA) kit (Zellbio Co., Germany).

\section{Data analysis}

All the data were reported as mean \pm SEM. Comparisons of the novel and familiar explorations (within-group comparisons) were analyzed using the paired Student's t-test. The behavioral data of the various groups (i.e., between-group comparisons) were compared using ANOVA followed by LSD posthoc test for multiple comparisons. Also, comparison of serum corticosterone levels were analyzed by ANOVA followed by Tukey's post-hoc test for multiple groups. A $P$-value of less than 0.05 was declared statistically significant. Ultimately, the calculations were performed using SPSS 21 (SPSS Inc. Chicago, IL, USA).

\section{Results}

\section{Total object exploration time and discrimination indexes (D1 and D2) in the OLT}

In the OLT test, an ANOVA followed by LSD post hoc test indicated no significant differences in $T 2[F(3$, 28) $=1.894, P>0.05$, Fig. $1 \mathrm{~A}$ ], in exploration times of familiar $(F)[F(3,28)=0.307, P>0.05]$ and new $(N)$ locations $[F(3,28)=2.375, P>0.05$, Fig. $1 B]$, in the main discrimination index (D2) $[F(3,28)=1.421$, $P>0.05$, Fig. $1 C]$ and in discrimination index (D1) $[F(3,28)=0.917, P>0.05$, Fig. $1 D]$. In the test phase of the OLT, the total object exploration times $(\mathrm{T} 2=\mathrm{F}+\mathrm{N})$ revealed no significant differences among the experimental groups (Fig. $1 \mathrm{~A}$ ), suggesting that crocin treatments did not improve locomotor activity in rats subjected to chronic restraint stress in the OLT.

As shown in Figure 1B, there were no differences between familiar $(\mathrm{F})$ and new $(\mathrm{N})$ locations exploration times as determined by the OLT test in the control $[\mathrm{t}(7)=-1.096, P>0.05]$, St.R $[\mathrm{t}(7)=0.910, P>0.05]$, St-R$\mathrm{C} 30[\mathrm{t}(7)=1.266, P>0.05]$ and St-R-C60 $[\mathrm{t}(7)=-0.648$, $P>0.05]$ groups, suggesting that restraint stress did not lead to rats' deteriorating ability to recognize novel locations as compared with their ability to recognize familiar locations. Moreover, new location exploration times as determined by the OLT showed significant differences between the St.R and St-RC30 groups $(P<0.05$, in both) as compared with that obtained for the control group (Fig. 1B). While the difference was significant $(P<0.05)$ between the St-RC60 group relative to the St-R group, indicating differences in the effects of different doses of crocin and the beneficial effect of $60 \mathrm{mg} / \mathrm{kg}$ of crocin on new location exploration times.

In the OLT test, all the groups recorded no significant differences in their main and auxiliary discrimination indices (D2 and D1, respectively) as compared with the control group (Figs. $1 C$ and $D$ ), indicating no 
A
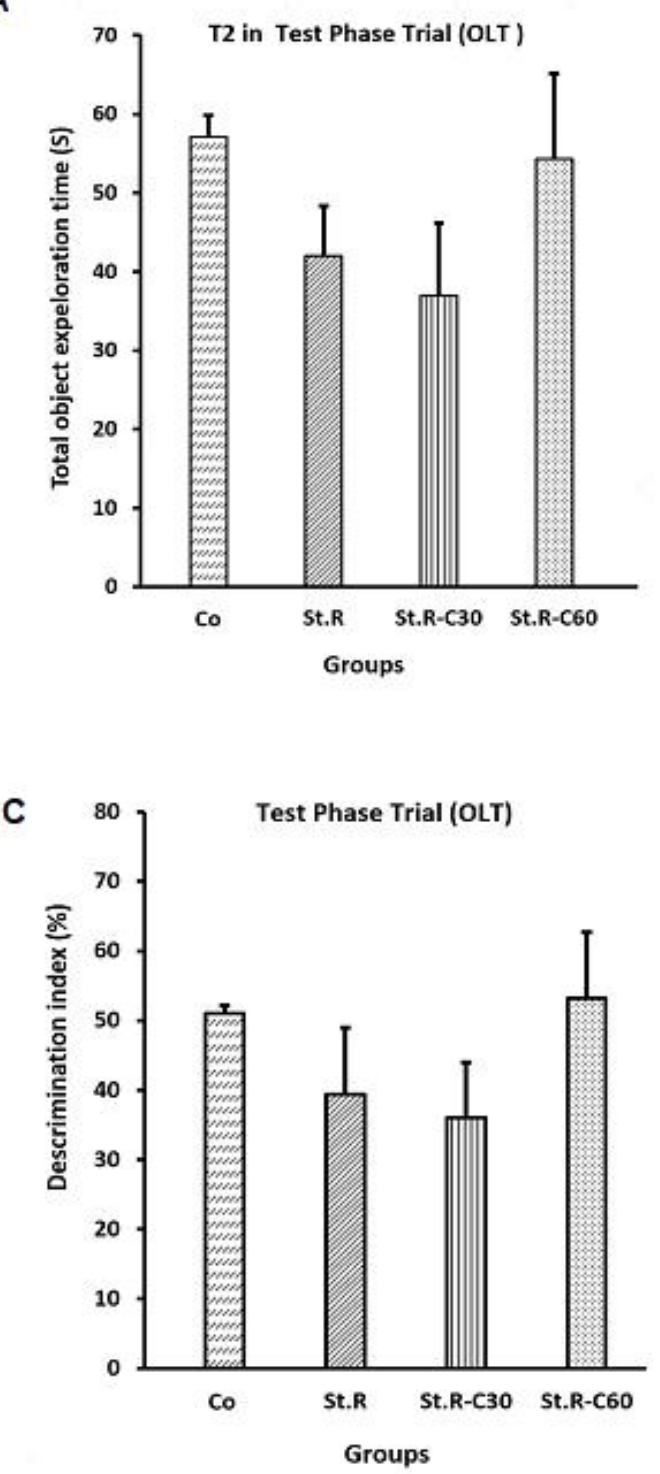

Test Phase Trial (OLT)
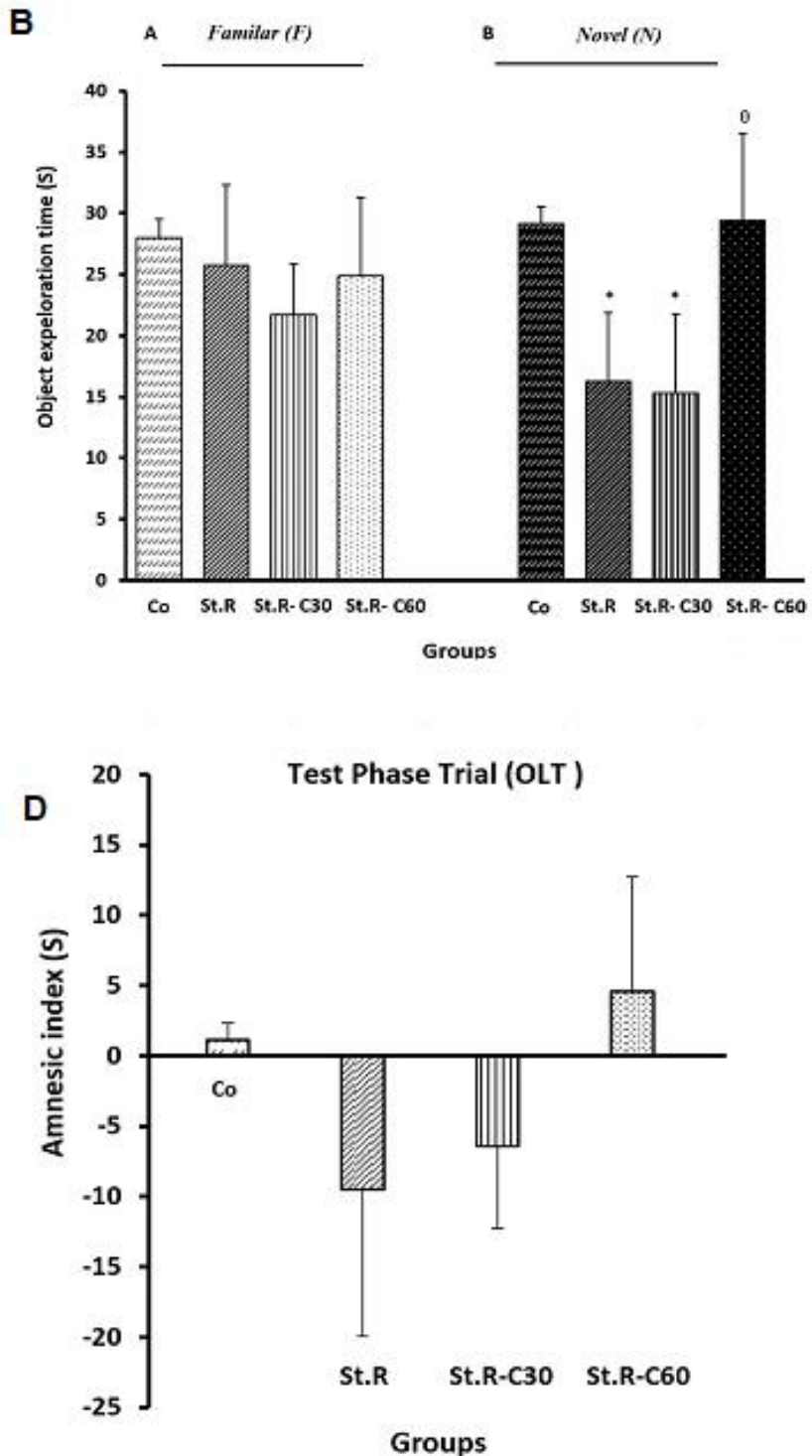

Fig.1. Effects of chronic restraint stress and two doses of crocin (30 or $60 \mathrm{mg} / \mathrm{kg}$ ) in the object location test (OLT): A) Total time of object exploration(s) for the test phase trial (T2) in the OLT; B) Exploration times of familiar and new locations (F and $\mathrm{N}$, respectively) in the test phase trial of the OLT; C) Values of the main discrimination index (D2) for the test phase trials of the OLT; and D) values of auxiliary discrimination index (D1) for the test phase trials of the OLT. Results are expressed as mean \pm SEM $(n=8)$. ${ }^{*} P<0.05$ compared to the control; ${ }^{\ominus} P<0.05$ compared to the stress group.

significantly decreased spatial memory across the experimental groups.

\section{Total object exploration time and discrimination indexes (D1 and D2) in the NOR}

In the NOR test, an ANOVA followed by LSD post hoc test indicated significant differences in $\mathrm{T} 2[\mathrm{~F}(3$, $28)=2.570, P<0.05$, Fig. $2 \mathrm{~A}]$, in exploration times of familiar $(F)[F(3,28)=0.653, P>0.05]$ and new $(N)$ locations $[F(3,28)=2.950, P<0.05$, Fig. $2 B])$, in the main discrimination index (D2) $[F(3,28)=0.624$, $P>0.05$, Fig. $2 \mathrm{C}$ ] and in discrimination index (D1) $[F(3,28)=0.471, P>0.05$, Fig. 2D).
Compared to the control group, the St.R group exhibited significantly $(P<0.05)$ decreased values for $\mathrm{T} 2$ in the test phase of NOR, indicating locomotor impairment in animals subjected to the chronic restraint stress (Fig. 2A). Furthermore, significant enhancements were observed in the T2 values obtained for the St.R-C30 and St.R-C60 groups $(P<0.05$ in both) relative to that of St.R (Fig. 2A), suggesting enhanced locomotor activity in both stressed groups as a result of daily injection of crocin (both 30 and $60 \mathrm{mg} / \mathrm{kg}$ doses) in the NOR test. However, no significant differences were found in T2 values between St.R-C30 and St.R-C60 groups (Fig. 
A
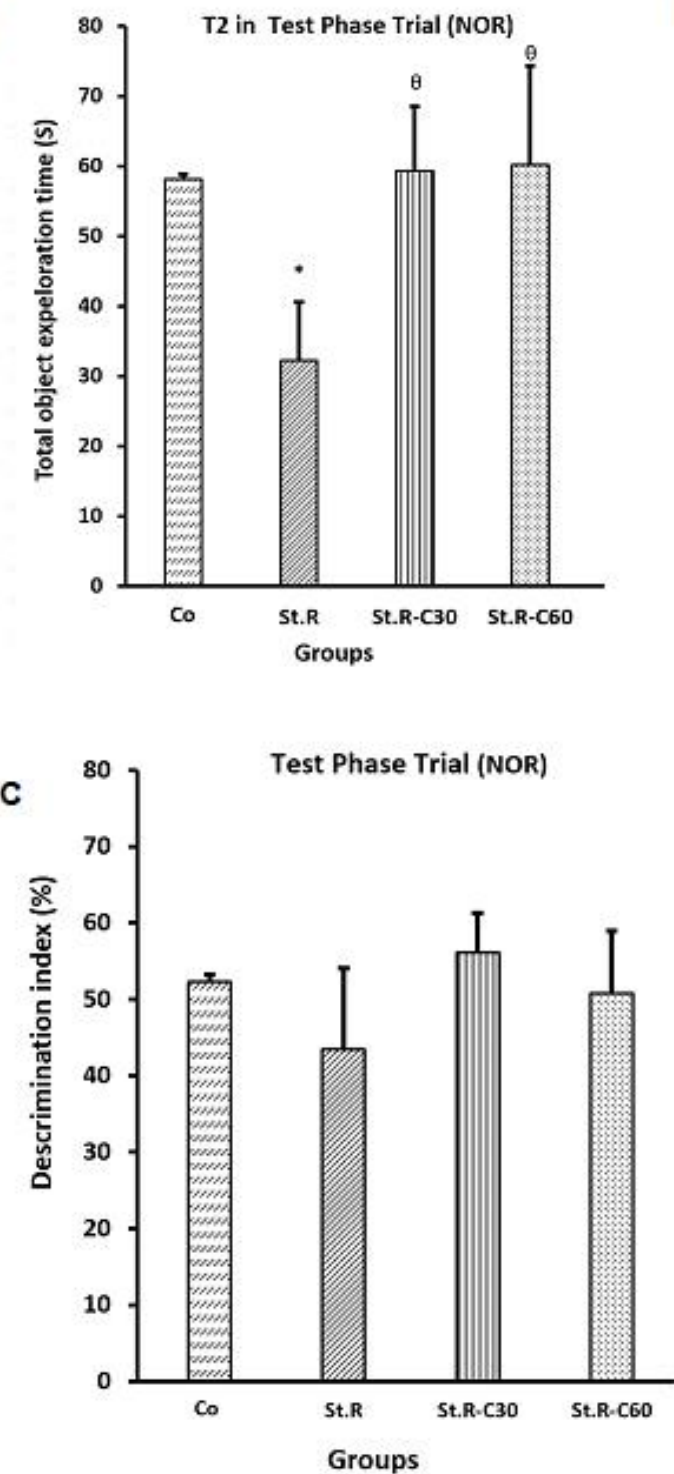

B

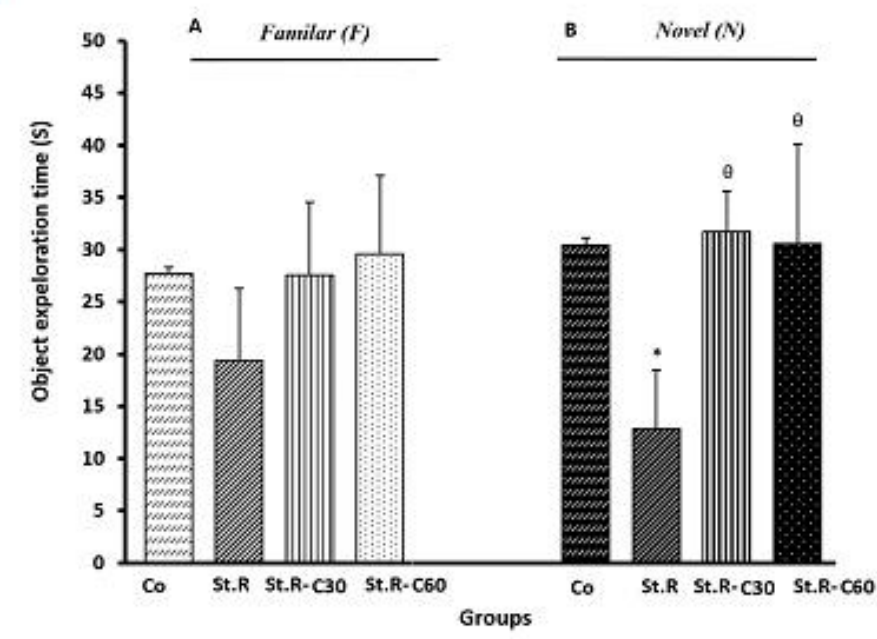

D

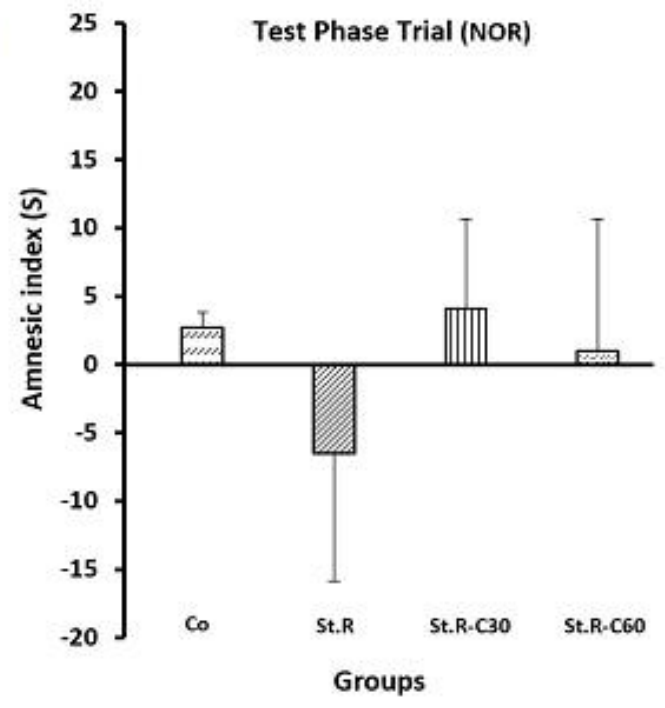

Fig.2. Effects of chronic restraint stress and two doses of crocin (30 or $60 \mathrm{mg} / \mathrm{kg}$ ) in the novel object recognition tests (NOR): A) Total time of object exploration(s) for the test phase trial (T2) in the NOR; B) Object exploration times of familiar and new objects ( $F$ and N, respectively) in the test phase trial of the NOR; C) value for the main discrimination index (D2) for the test phase trials of the NOR; D) value of the auxiliary discrimination index (D1) for the test phase trials of the NOR. Results are expressed as mean \pm SEM. ${ }^{*} P<0.05$ compared to the control; ${ }^{\ominus} P<0.05$ compared to the stress group.

2A).

As shown in Figure 2B, there were no differences between familiar $(\mathrm{F})$ and new $(\mathrm{N})$ locations exploration times as determined by the NOR test in the control $[\mathrm{t}(7)=-2.299, P>0.05]$, St.R $[\mathrm{t}(7)=0.694, P>0.05]$, St-R$\mathrm{C} 30[\mathrm{t}(7)=-0.734, P>0.05]$ and St-R-C60 $[\mathrm{t}(7)=-0.120$, $P>0.05$ ] groups, indicating that restraint stress did not affect novel object recognition when compared with familiar object recognition, in the NOR test. Also, comparison of new object exploration times of NOR showed a significant $(P<0.05)$ decrease in the St.R group relative to new object exploration time in the control group (Fig. 2B). However, significant enhancements were observed in the St-R-C30 and St-R-C60 groups $(P<0.05$, in both) when compared with the St-R group. This suggested that crocin (at doses of 30 and $60 \mathrm{mg} / \mathrm{kg}$ ) affected the ability of the restraint stress groups to recognize novel objects.

The main discrimination and auxiliary indexes (D2 and D1) in the NOR test showed no significant decreases across the experimental groups (Figs. 2C and $D$ ) indicating that different doses of crocin were not able to improve cognitive memory.

\section{Changes in serum corticosterone levels}

An ANOVA followed by Tukey's post-hoc test 


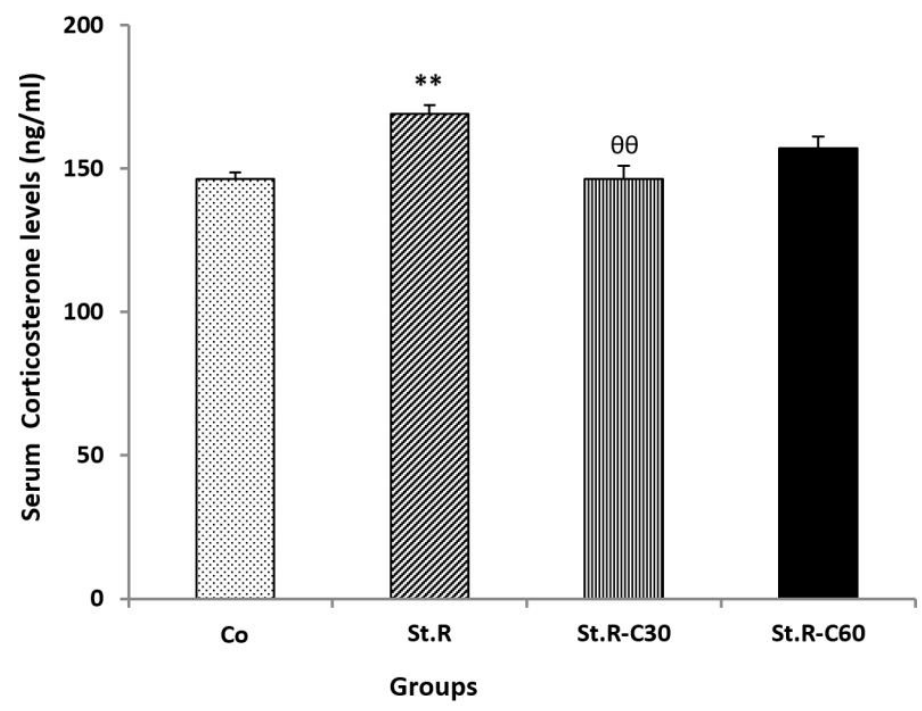

Fig.3. Effects of chronic restraint stress and different doses of crocin (30 or $60 \mathrm{mg} / \mathrm{kg}$ ) on the serum corticosterone (CORT) levels $(\mathrm{ng} / \mathrm{ml})$ in different groups $(\mathrm{n}=8)$. The results are expressed as mean $\pm \operatorname{SEM}(\mathrm{n}=8) .{ }^{* *} P<0.01$ compared to the control group; ${ }^{\ominus \ominus} P<0.01$ compared to the stress group.

indicated significant differences in serum corticosterone levels $[F(3,28)=8.876, P<0.001$, Fig. 3]. The St.R group showed significant $(P<0.01)$ increases in serum corticosterone level (Fig. 3). As shown in Figure 3, corticosterone levels decreased significantly $(P<0.01)$ only in the St.R-C30 group when compared with the St.R group, indicating the protective effect of crocin at a dosage of $30 \mathrm{mg} / \mathrm{kg}$ on serum corticosterone as the main stress hormone in stressed rats.

\section{Discussion}

The effects of different crocin doses (low and high doses) were investigated on locomotor activity, recognition of new conditions, novel object recognition/ location memories and serum corticosterone level in rats subjected to chronic restraint stress using the novel object recognition and object location tests, respectively.

The present OLT findings showed that chronic restraint stress reduced the ability to recognize new conditions, but not locomotor activity or object location memory (Fig. 1). However, the NOR findings revealed significant impairments as a result of chronic restraint stress in locomotor activity and recognition of new condition, but not in object recognition memory (Fig. 2). Whereas, Varty et al. (2000) demonstrated that isolation stress increases exploratory behaviors, some reports indicated that other types of chronic stress destroy both object recognition and cognitive memory (Maeng and Shors, 2013; Oei et al., 2006; Ranjbar et al., 2016; Simoens et al., 2007). In addition, these findings were not consistent with those reported previously in a study of animal models of chronic isolation stress that indicated severely impaired locomotor activity, reduced exploration time and impaired cognitive as well as spatial memories as revealed by OLT and by NOR test in particular (Khani et al., 2018). It seems that the brain impairments observed in the chronic restraint stressed group were partly mediated probably by elevated serum corticosterone levels (Fig. 3), an idea that is confirmed elsewhere (Conrad, 2010; Khani et al., 2018). It is well known that stress is associated with elevated secretion of glucocorticoids and that some brain functions are probably impaired due to abnormally high glucocorticoid levels or oxidative stress (Ghadrdoost et al., 2011).

Based on the findings of the present study, only the NOR test results indicated increase in locomotor activity and exploration time in the stressed groups receiving crocin doses of 30 and $60 \mathrm{mg} / \mathrm{kg}$ when compared with the stressed group receiving no crocin (Fig. 2). However, the OLT test results revealed no significant differences in locomotor activity while exploration time was significantly improved only in the St.R-C60 group in comparison with the stressed group (Fig. 1). It seems that the NOR test was more sensitive than the OLT to slight changes in this regard. In this way, some studies reported that crocin 
may increase or decrease locomotor activity or have no effect on it (Amin et al., 2015; Karami et al., 2013). Additionally, saffron extract changed the locomotor activity dose dependently in normal animals (Hosseinzadeh and Noraei, 2009). Moreover, neither NOR test nor OLT test showed improvements in either type of memory. It may be noted that previous reports indicated that crocin alone was able to improve both learning and memory (Abe and Saito, 2000; Pitsikas and Sakellaridis, 2006; Pitsikas et al., 2007).

Using the same behavioral tests (NOR and OLT), Khani et al. (2018) also reported that both crocin doses had been able to improve locomotor activity, exploration time and cognitive and spatial memories in animals subjected to chronic isolation stress. Crocin seems to have a greater influence on improving brain functions in the animals subjected to chronic isolation stress than in those under chronic restraint stress in the current study. This difference might explain the differences in the NOR and OLT results observed between the chronic restraint stress group in the current study and the chronic isolation stress in Khani's study. Thus, it might be suggested that the type of imposed stress, plays an important role in the effects of treatment with crocin on brain functions by similar behavior tests. McMorran et al. (2006) indicated that type of stress plays a role in such brain functions as mood, memory, attention and cognition. On the other hand, Dastgerdi et al. (2017) employed passive avoidance as a type of behavioral test different from the ones used in the current study (the NOR and OLT tests) but with similar crocin treatments, only to find different effects of crocin on memory processing. This also provides support for the hypothesis that the type of behavioral test used plays a role in the effects of crocin treatment detected on brain functions. Other studies also reported memory impairments due to chronic stress in such varied spatial memory tasks as the Morris water, (Venero et al., 2002), radial-arm (Atsak et al., 2016) and $Y$ mazes (Hao et al., 2014). Generally, it is noticeable that various effects of stress and crocin on different behavioral tests are not only affected by the type of stress but also by the type of task behavior designated, induced stress time and duration, gender and age (Dastgerdi et al., 2017; Diamond et al., 2007; Ennaceur, 2010; Joëls et al., 2006; Ranjbar et al., 2016; Sandi and Pinelo-Nava, 2007; Wolf, 2008).
Regarding the data on corticosterone level, it was found to decrease only in the St.R-C30 group when compared with the stressed group, indicating the protective effect of $30 \mathrm{mg} / \mathrm{kg}$ of crocin on serum corticosterone levels as the main stress hormone in the chronic restraint stressed rats. Some studies also reported that crocin could decrease the corticosterone response to chronic stress (Ghadrdoost et al., 2011). It seems that the beneficial effects of crocin are not only dose-dependent but also related to stress type and the behavioral tests employed. In addition, although the serum corticosterone level was decreased just following the low dose $(30 \mathrm{mg} / \mathrm{kg})$, the improvement of total exploration times were observed after the high dose $(60 \mathrm{mg} / \mathrm{kg})$ in both OLT and NOR tests. It seems that effect of high dose of crocin did not dependent to only corticosterone levels on total exploration times in the OLT and NOR tests. Therefore, it is possible that crocin may influent some neurotransmitters such as adrenaline, dopamine, serotonin (Ettehadi et al., 2013; Hosseinzadeh et al., 2004), acetyl choline, GABA and glutamate (Anwar et al., 2017; Ettehadi et al., 2013; Hosseinzadeh and Sadeghnia, 2007). It is possible that even different neurotransmitters may involve in different doses of crocin (Anwar et al., 2017; Hosseinzadeh and Sadeghnia, 2007). However, neither the exact mechanisms nor the anatomical sites involved in the inhibitory action of crocin on corticosterone levels are yet known, awaiting further research. Moreover, such other variables as stress and memory biomarkers might be of influence in the responsiveness of behavioral tests to crocin treatment. In other words, not only corticosterone levels but other mechanisms such as the antioxidant/oxidant balance (Abe and Saito, 2000; Asdaq and Inamdar, 2010; Bickford et al., 2000; Erdemli, 2018; Ochiai et al., 2004; Papandreou et al., 2006), free radical scavenging (Abe and Saito, 2000), modulation of NMDA receptor functions (Abe and Saito, 2000), neurotransmitter release (Ettehadi et al., 2013; Hosseinzadeh et al., 2008), brain-derived neurotrophic factor (BDNF) and gene expression (Dorri et al., 2015; Rezai et al., 2018) might be involved in changes in the brain functions as detected (e.g., by behavioral tests) in crocin treatment.

It is worth noting that the current study is novel in several respects when compared with Khani et al. (2018) and Dastgerdi et al. (2017) among others. These include stress type (chronic restraint stress vs 
chronic isolation stress), the behavioral tests employed (NOR and OLT used to evaluate two different types of memory with no external motivation and degree of stress in animal laboratories vs passive avoidance with shock induction) and the different doses of crocin used in the treatments (Ghadrdoost et al., 2011; Khani et al., 2018 ).

\section{Conclusion}

It was shown in this study that locomotor activity and exploration time, rather than the different novel object recognition and object location memories, were almost impaired by chronic restraint stress. Moreover, exploration time was found to be affected by both low and high crocin doses in the NOR test but by only the high crocin dose in the OLT test. Thus, the beneficial effects of low and high doses of crocin were detected by some of the variables in each of the different behavioral tests under stress conditions. More specifically, the NOR, as a cognitive test, was found more sensitive to crocin doses than was the OLT, as a spatial test. Finally, although neither of the two memories examined showed changes under crocin treatment, it is important to reiterate the mechanism(s) underlying the role of crocin in memory improvement.

\section{Acknowledgments}

The authors would like to Hajaralsadat Hosseini Dastgerdi1for their valuable assistance. This work was supported by grants from Isfahan University of Medical Sciences, Isfahan, Iran.

\section{Conflict of interest}

There are no conflicts of interest that have been reported by the authors or by any individuals in control of the content of this article.

\section{References}

Abe K, Saito H. Effects of saffron extract and its constituent crocin on learning behaviour and long-term potentiation. Phytother Res 2000; 14: 149-52. https://doi.org/ 10.1002/(SICI)1099-1573(200005)14:3<149::AIDPTR665>3.0.CO;2-5

Ainge JA, Heron-Maxwell C, Theofilas $\mathrm{P}$, Wright $\mathrm{P}$, de $\mathrm{Hoz}$ $L$, Wood ER. The role of the hippocampus in object recognition in rats: examination of the influence of task parameters and lesion size. Behav Brain Res 2006; 167: 183-95. https://doi.org/10.1016/j.bbr.2005.09.005

Akkerman S, Blokland A, Reneerkens O, van Goethem NP, Bollen E, Gijselaers HJ, et al. Object recognition testing: methodological considerations on exploration and discrimination measures. Behav Brain Res 2012; 232: 335-47. https://doi.org/10.1016/j.bbr.2012.03.022

Amin B, Nakhsaz A, Hosseinzadeh $H$. Evaluation of the antidepressant-like effects of acute and sub-acute administration of crocin and crocetin in mice. Avicenna J Phytomed 2015; 5: 458-468.

Anwar N, Ahmed NZ, Shahida T, Kabiruddin T, Aslam H. The role of mufarrehat (exhilarants) in the management of depression: an evidence based approach. J Psychiatry 2017; 20: 2. https://doi.org/10.4172/23785756.1000420

Asdaq SM, Inamdar MN. Potential of crocus sativus (saffron) and its constituent, crocin, as hypolipidemic and antioxidant in rats. Appl Biochem Biotechnol 2010; 162: 358-72. https://doi.org/10.1007/s12010-009-8740-7

Atsak P, Guenzel FM, Kantar-Gok D, Zalachoras I, Yargicoglu P, Meijer OC, et al. Glucocorticoids mediate stress-induced impairment of retrieval of stimulusresponse memory. Psychoneuroendocrinology 2016; 67: 207-15. https://doi.org/10.1016/j.psyneuen.2016.02. 006

Bandegi AR, Rashidy-Pour A, Vafaei AA, Ghadrdoost B. Protective effects of crocus sativus I. extract and crocin against chronic-stress induced oxidative damage of brain, liver and kidneys in rats. Adv Pharm Bull 2014; 4: 493-99.

Bickford PC, Gould T, Briederick L, Chadman K, Pollock A, Young $D$, et al. Antioxidant-rich diets improve cerebellar physiology and motor learning in aged rats. Brain Res 2000; 866: 211-7. https://doi.org/10.1016/S0006-8993 (00)02280-0

Blanchard RJ, McKittrick CR, Blanchard DC. Animal models of social stress: effects on behavior and brain neurochemical systems. Physiol Behav 2001; 73: 261 71. https://doi.org/10.1016/S0031-9384(01)00449-8

Brodziak A, Kolat E, Rozyk-Myrta A. In search of memory tests equivalent for experiments on animals and humans. Med Sci Monit 2014; 20: 2733-39. https://doi.org/10.12659/MSM.891056

Clark RE, Zola SM, Squire LR. Impaired recognition memory in rats after damage to the hippocampus. $J$ Neurosci 2000; 20: 8853-60. https://doi.org/10.1523/ JNEUROSCI.20-23-08853.2000

Conrad CD. A critical review of chronic stress effects on spatial learning and memory. Prog Neuropsychopharmacol Biol Psychiatry 2010; 34: 74255. https://doi.org/10.1016/j.pnpbp.2009.11.003

Dastgerdi AH, Radahmadi M, Pourshanazari AA, Dastgerdi $\mathrm{HH}$. Effects of crocin on learning and memory in rats under chronic restraint stress with special focus on the hippocampal and frontal cortex corticosterone levels. Adv Biomed Res 2017; 6: 157. https://doi.org/10.4103 /abr.abr_107_17

Diamond DM, Campbell AM, Park CR, Halonen J, Zoladz PR. The temporal dynamics model of emotional memory processing: A synthesis on the neurobiological basis of stress-induced amnesia, flashbulb and traumatic memories, and the yerkes-dodson law. Neural 
Plast 2007; 2007: 1-33. https://doi.org/10.1155/2007/ 60803

Dorri SA, Hosseinzadeh H, Abnous K, Hasani FV, Robati RY, Razavi BM. Involvement of brain-derived neurotrophic factor (bdnf) on malathion induced depressive-like behavior in subacute exposure and protective effects of crocin. Iran J Basic Med Sci 2015; 18: 958-66.

Drumond LE, Mourão FA, Leite HR, Abreu RV, Reis HJ, Moraes MF, et al. Differential effects of swimming training on neuronal calcium sensor-1 expression in rat hippocampus/cortex and in object recognition memory tasks. Brain Res Bull 2012; 88: 385-91. https://doi.org/10.1016/j.brainresbull.2012.04.005

Ennaceur A. One-trial object recognition in rats and mice: methodological and theoretical issues. Behav Brain Res 2010; 215: 244-54. https://doi.org/10.1016/j.bbr.2009. 12.036

Erdemli ME. The protective role of crocin on acrylamideinduced oxidative neurotoxicity in rat. $\mathrm{J}$ Cell Neurosci Oxid Stress 2018; 10.

Ettehadi H, Mojabi SN, Ranjbaran M, Shams J, Sahraei H, Hedayati $M$, et al. Aqueous extract of saffron (crocus sativus) increases brain dopamine and glutamate concentrations in rats. J Behav Brain Sci 2013; 3: 3159. https://doi.org/10.4236/jbbs.2013.33031

Ghadrdoost B, Vafaei AA, Rashidy-Pour A, Hajisoltani R, Bandegi AR, Motamedi $F$, et al. Protective effects of saffron extract and its active constituent crocin against oxidative stress and spatial learning and memory deficits induced by chronic stress in rats. Eur $\mathrm{J}$ Pharmacol 2011; 667: 222-9. https://doi.org/10.1016/ j.ejphar.2011.05.012

Gonda S, Parizsa P, Surányi G, Gyémánt G, Vasas G. Quantification of main bioactive metabolites from saffron (crocus sativus) stigmas by a micellar electrokinetic chromatographic (mekc) method. J Pharm Biomed Anal 2012; 66: 68-74. https://doi.org/10.1016 /j.jpba.2012.03.002

Hao L, Huang H, Gao J, Marshall C, Chen Y, Xiao M. The influence of gender, age and treatment time on brain oxidative stress and memory impairment induced by dgalactose in mice. Neurosci Lett 2014; 571: 45-9. https://doi.org/10.1016/j.neulet.2014.04.038

Hosseinzadeh H, Karimi GH, Niapoor M. Antidepressant effects of crocus sativus stigma extracts and its constituents, crocin and safranal, in mice. J Med Plant 2004; 3: 48-58. https://doi.org/10.17660/ActaHortic. 2004.650 .54

Hosseinzadeh $\mathrm{H}$, Noraei NB. Anxiolytic and hypnotic effect of crocus sativus aqueous extract and its constituents, crocin and safranal, in mice. Phytother Res 2009; 23: 768-74. https://doi.org/10.1002/ptr.2597

Hosseinzadeh $H$, Sadeghnia HR. Protective effect of safranal on pentylenetetrazol-induced seizures in the rat: Involvement of gabaergic and opioids systems. Phytomedicine 2007; 14: 256-62. https://doi.org/10. 1016/j.phymed.2006.03.007

Hosseinzadeh H, Sadeghnia HR, Rahimi A. Effect of safranal on extracellular hippocampal levels of glutamate and aspartate during kainic acid treatment in anesthetized rats. Planta Medica 2008; 74: 1441-5. https://doi.org/10.1055/s-2008-1081335

Hosseinzadeh $\mathrm{H}$, Ziaei $\mathrm{T}$. Effects of crocus sativus stigma extract and its constituents, crocin and safranal, on intact memory and scopolamine-induced learning deficits in rats performing the morris water maze task. $\mathrm{J}$ Med Plant 2006; 3: 40-50.

Joëls M, Pu Z, Wiegert O, Oitzl MS, Krugers HJ. Learning under stress: how does it work? Trends Cogn Sci 2006; 10: 152-8. https://doi.org/10.1016/j.tics.2006.02.002

Karami M, Bathaie SZ, Tiraihi T, Habibi-Rezaei M, Arabkheradmand J, Faghihzadeh S. Crocin improved locomotor function and mechanical behavior in the rat model of contused spinal cord injury through decreasing calcitonin gene related peptide (cgrp). Phytomedicine 2013; 21: 62-7. https://doi.org/10.1016/j.phymed. 2013.07.013

Khani F, Radahmadi M, Alaei H, Jafari E. Effects of crocin on cognitive and spatial memories in rats under chronic isolation stress. Physiol Pharmacol 2018; 22: 254-68.

Maeng LY, Shors TJ. The stressed female brain: neuronal activity in the prelimbic but not infralimbic region of the medial prefrontal cortex suppresses learning after acute stress. Front Neural Circuits 2013; 7: 198. https://doi.org/10.3389/fncir.2013.00198

McMorris T, Swain J, Smith M, Corbett J, Delves S, Sale C, et al. Heat stress, plasma concentrations of adrenaline, noradrenaline, 5-hydroxytryptamine and cortisol, mood state and cognitive performance. Int J Psychophysiol 2006; 61: 204-15. https://doi.org/10.1016/j.ijpsycho 2005.10.002

Mohammadi HS, Goudarzi I, Lashkarbolouki T, Abrari K, Salmani ME. Chronic administration of quercetin prevent spatial learning and memory deficits provoked by chronic stress in rats. Behav Brain Res 2014; 270: 196-205. https://doi.org/10.1016/j.bbr.2014.05.015

Ochiai T, Ohno S, Soeda S, Tanaka H, Shoyama Y, Shimeno $H$. Crocin prevents the death of rat pheochromyctoma (PC-12) cells by its antioxidant effects stronger than those of $\alpha$-tocopherol. Neurosci Lett 2004; 362: 61-4. https://doi.org/10.1016/j.neulet. 2004.02.067

Oei NY, Everaerd WT, Elzinga BM, van Well S, Bermond B. Psychosocial stress impairs working memory at high loads: an association with cortisol levels and memory retrieval. Stress 2006; 9: 133-41. https://doi.org/ 10.1080/10253890600965773

Papandreou MA, Kanakis CD, Polissiou MG, Efthimiopoulos $S$, Cordopatis $P$, Margarity $M$, et al. Inhibitory activity on amyloid- $\beta$ aggregation and antioxidant properties of crocus sativus stigmas extract and its crocin constituents. J Agric Food Chem 2006; 54: 8762-8. https://doi.org/10.1021/jf061932a

Patchev VK, Patchev AV. Experimental models of stress. Dialogues Clin Neurosci 2006; 8: 417.

Pitsikas N, Boultadakis A, Georgiadou G, Tarantilis PA, Sakellaridis N. Effects of the active constituents of 
crocus sativus L., crocins, in an animal model of anxiety. Phytomedicine 2008; 15: 1135-9. https://doi. org/10.1016/j.phymed.2008.06.005

Pitsikas N, Sakellaridis N. Crocus sativus L. Extracts antagonize memory impairments in different behavioural tasks in the rat. Behav Brain Res 2006; 173: 112-5. https://doi.org/10.1016/j.bbr.2006.06.005

Pitsikas N, Zisopoulou S, Tarantilis PA, Kanakis CD, Polissiou MG, Sakellaridis N. Effects of the active constituents of crocus sativus I., crocins on recognition and spatial rats' memory. Behav Brain Res 2007; 183: 141-6. https://doi.org/10.1016/j.bbr.2007.06.001

Ranjbar H, Radahmadi M, Alaei H, Reisi P, Karimi S. The effect of basolateral amygdala nucleus lesion on memory under acute, mid and chronic stress in male rats. Turk J Med Sci 2016; 46: 1915-25. https://doi.org/10.3906/sag-1507-7

Rezai M, Mahmoodi M, Kaeidi A, Karimabad MN, Khoshdel A, Hajizadeh MR. Effect of crocin carotenoid on bdnf and creb gene expression in brain ventral tegmental area of morphine treated rats. Asian Pac J Trop Biomed 2018; 8: 387-93. https://doi.org/10.4103/22211691.239426

Roozendaal B, Barsegyan A, Lee S. Adrenal stress hormones, amygdala activation, and memory for emotionally arousing experiences. Prog Brain Res 2007; 167: 79-97. https://doi.org/10.1016/S00796123(07)67006-X

Sandi C, Pinelo-Nava MT. Stress and memory: behavioral effects and neurobiological mechanisms. Neural plasticity 2007; 2007: 1-20. https://doi.org/10.1155/ $2007 / 78970$

Sik A, van Nieuwehuyzen P, Prickaerts J, Blokland A. Performance of different mouse strains in an object recognition task. Behav Brain Res 2003; 147: 49-54. https://doi.org/10.1016/S0166-4328(03)00117-7

Silvers JM, Harrod SB, Mactutus CF, Booze RM. Automation of the novel object recognition task for use in adolescent rats. J Neurosci Methods 2007; 166: 99103. https://doi.org/10.1016/j.jneumeth.2007.06.032

Simoens VL, Istok E, Hyttinen S, Hirvonen A, Naatanen R, Tervaniemi M. Psychosocial stress attenuates general sound processing and duration change detection. Psychophysiology 2007; 44: 30-8. https://doi.org/ 10.1111/j.1469-8986.2006.00476.x

Varty GB, Paulus MP, Braff DL, Geyer MA. Environmental enrichment and isolation rearing in the rat: Effects on locomotor behavior and startle response plasticity. Biol Psychiatry 2000; 47: 864-73. https://doi.org/10.1016/ S0006-3223(99)00269-3

Venero C, Tilling T, Hermans-Borgmeyer I, Schmidt R, Schachner M, Sandi C. Chronic stress induces opposite changes in the mrna expression of the cell adhesion molecules ncam and L1. Neuroscience 2002; 115: 1211-9. https://doi.org/10.1016/S0306-4522(02)00543-2

Waters KA, Stean TO, Hammond B, Virley DJ, Upton N, Kew JN, et al. Effects of the selective 5-HT7 receptor antagonist SB-269970 in animal models of psychosis and cognition. Behav Brain Res 2012; 228: 211-8. https://doi.org/10.1016/j.bbr.2011.12.009

Wolf OT. The influence of stress hormones on emotional memory: relevance for psychopathology. Acta Psychologica 2008; 127: 513-31. https://doi.org/ 10.1016/j.actpsy.2007.08.002

Wu L, Lu Y, Jiao Y, Liu B, Li S, Li Y, et al. Paternal psychological stress reprograms hepatic gluconeogenesis in offspring. Cell Metab 2016; 23: 73543. https://doi.org/10.1016/j.cmet.2016.01.014 